\title{
A Random Distribution Reacting Mixing Layer Model
}

Richard A. Jones

Rensselaer Polytechnic Institute

Troy, New York

January 1994

Prepared for

Lewis Research Center

Under Contract NCC-3-213

\section{N/SA




\title{
A RANDOM DISTRIBUTION REACTING MIXING LAYER MODEL
}

\author{
Richard A. Jones* \\ Rensselaer Polytechnic Institute \\ Troy, New York 12181
}

\section{SUMMARY}

A methodology for simulation of molecular mixing, and the resulting velocity and temperature fields has been developed. The ideas are applied to the flow conditions present in the NASA Lewis Planar Reacting Shear Layer (PRSL) facility, and results compared to experimental data. A gaussian transverse turbulent velocity distribution is used in conjunction with a linearly increasing time scale to describe the mixing of different regions of the flow. Equilibrium reaction calculations are then performed on the mix to arrive at a new species composition and temperature. Velocities are determined through summation of momentum contributions. The analysis indicates a combustion efficiency of the order of 80 percent for the reacting mixing layer, and a turbulent Schmidt number of $2 / 3$. The success of the model is attributed to the simulation of large-scale transport of fluid. The favorable comparison shows that a relatively quick and simple PC calculation is capable of simulating the basic flow structure in the reacting and non-reacting shear layer present in the facility given basic assumptions about turbulence properties.

\section{INTRODUCTION}

This report summarizes the theory behind mixing layer simulations created through the use of a mixing simulation program and the NASA Lewis CETPC Chemical Equilibrium Composition program, ${ }^{1}$ and presents some initial results. The mixing program, entitled MYV.FOR, was created to simulate the velocity, thermal, and species distributions in the NASA Lewis Research Center Planar Reacting Shear Layer (PRSL) facility. ${ }^{2}$ In evaluation of the modeling, experimental thermocouple and Laser Doppler Velocimetry (LDV) data is used. 3,4 MYV.FOR is not a fluid dynamics program, but rather a methodology for simulation of molecular mixing. A transport model based on transverse turbulent velocity profiles and integral time scales is used to simulate the mixing of molecules in the flowfield. Through conservation of momentum, a new velocity distribution is determined. The mix is assumed to react and convect to a downstream location. The new composition is the initial condition for subsequent mixing, reaction, and convection at the downstream location.

Motivation for the current effort lies in the large amount of time required to generate a full Navier-Stokes reacting flow simulation of the mixing layer. It is desired to develop and demonstrate a simple model which can run in minutes on a typical desk top computer. Advantages include both the timeliness of the results, and the insight into the turbulent transport gained.

Previous work has been done for the reacting shear layer configuration, both experimentally and computationally, by many investigators. Because the emphasis of this work is on the simplicity and speed of the model, only simple, empirically based works are mentioned here. Konrad ${ }^{5}$ measured the probability density function (PDF) of molecular constituents in a mixing layer formed from two dissimilar streams. He computed a product PDF based on the two freestream reactant PDF's for a theoretical reacting mixing layer. For comparison purposes, he compared that result with that resulting from using only mean reactant profiles, which is similar to the current effort. He found that use of the PDF model lowered temperatures and product fraction significantly below the equilibrium calculation.

\footnotetext{
* Summer Student Intern at NASA Lewis Research Center.
} 
Wallace $^{6}$ used Konrad's non-reacting PDF's in a similar calculation, with the goal of simulating his experimentally obtained reacting mixing layer thermal profiles. The computational profiles were, in general, higher in temperature, shifted in position, and over-predicted diffusion.

In contrast, the current approach uses the transverse velocity PDF in an attempt to model time averaged momentum diffusion. An assumed Schmidt number is used to model molecular mixing. Then, an equilibrium calculation is performed to arrive at post-reaction molecular compositions, temperatures, and densities. Momentum mixing is performed as well, and mean streamwise velocity profiles generated.

\section{COMPUTATIONAL DOMAIN}

A schematic of the experimental facility is shown in Fig. 1. The upper stream typically flows at a Mach number of 0.4 . For non-reacting tests, air is used in both streams, while for the reacting mixing layer studies, the upper stream consists of hydrogen diluted with nitrogen. The lower stream for both reacting and nonreacting studies is heated air flowing at a Mach number of 0.7 . The test section is approximately $40 \mathrm{~cm}$ in the streamwise direction, $10 \mathrm{~cm}$ in the vertical direction, and $20 \mathrm{~cm}$ in width.

For computational purposes, the PRSL test section is divided into 400 transverse elements. Experimentally, the reacting mixing layer is found to impinge the top of the test section at far downstream positions, due to the large growth angle and upward slant of the layer. The computational method simulates transverse fluid movement from each freestream into the mixing layer. Thus, the computational domain extends beyond the dimensions of the actual test section to avoid difficulties with the statistical mixing procedure. Because of the large transverse influence distance at these downstream locations, the computational domain is actually four times the height of the test section. Each computational element is initially $1.016 \mathrm{~mm}$ in height. The lengths of the computational elements are determined by the streamwise convection distance which will be discussed further.

Since the facility is designed to impose a neutral axial pressure gradient through adjustable walls, and experimental data shows that this is reasonably accomplished, constant pressure combustion calculations are used. Thus, density changes considerably with heat release, by more than a factor of three near stoichiometry. Because no axial acceleration of the fluid is observed, and the fluid is constrained to a constant width in the cross-stream direction, all growth necessary for the conservation of mass is assumed to take place in the transverse direction; that is, the initial height of the fluid elements described above is allowed to increase to account for fluid expansion.

\section{COMPUTATIONAL METHOD}

The method can be summarized as a statistical molecular mixing simulation followed by equilibrium combustion and convection downstream of reaction products. The important parameters governing the mixing are the transverse turbulent velocity probability density function (PDF) and the integral time scale $I$ of the turbulent flowfield. Each fluid element is allowed to disperse and travel in the transverse direction. The relative amount of each element travelling at a given transverse velocity is determined by the PDF. The transverse velocity, coupled with a time scale, determines the cross-stream distance travelled by all portions of each fluid element. The same time scale is used in conjunction with the average streamwise velocity to determine downstream convection distance.

For the transverse turbulent velocity a gaussian PDF is used throughout the presented simulations. The selection of this PDF function is discussed in the Computational Parameters section. Thus, for zero mean 
transverse velocity during a mixing simulation, the majority of a given fluid element will have zero cross-stream velocity, while decreasing amounts will have both positive and negative transverse velocities of increasing magnitude.

The integral time scale $I$ should be thought of as a "coherence time" for fluid eddies during travel. Each PDF weighted portion of a fluid element is assumed to travel for time $I$ before mixing with other arriving fluid elements. Empirically, we expect the integral time scale for the mixing layer to increase linearly with streamwise distance. Selection of $I$, and the effect on computational layer growth rate, is discussed in the Computational Parameters section.

Computationally, we look at the contributions to a point of interest from all other elements in the flow. Thus, we shift here from a description of the dispersion and travel of a given fluid element to a calculation of contributions from all elements to a point of interest. Figure 2 shows a schematic of three contributing elements to a computational point and their travel distances. Elements $\mathrm{A}, \mathrm{B}$, and $\mathrm{C}$ are transverse distances $\mathrm{y}(\mathrm{A}), \mathrm{y}(\mathrm{B})$, and $\mathrm{y}(\mathrm{C})$ from the element of interest $\mathrm{D}$. There exists a local integral time scale $I$ over which fluid may travel. The combination of transverse distance and travel time produce a required transverse velocity for contribution of $\mathrm{A}, \mathrm{B}$, or $\mathrm{C}$ to $\mathrm{D}$ as

$$
V_{j}=\frac{y_{j}}{I}
$$

where $\mathrm{V}$ is the transverse velocity and the index " $j "$ represents transverse fluid elements. Using the gaussian cross-stream velocity PDF for A, B, and C, we can determine the portion of each element which will possess the required transverse velocity to travel to $\mathrm{D}$ in time $I$ through

$$
\frac{1}{\sqrt{2 \pi V_{r m s}^{\prime}}} e^{-\left(V^{\prime}-\bar{V}\right)^{2} / 2 V_{r m s}^{\prime 2}}
$$

The product of the number density in molecules/cc and volume of the contributing element are multiplied by this weighting to determine the number of molecules arriving at D. The enthalpy of each contributing fluid element is summed ${ }^{7}$ to arrive at a total number and enthalpy of each molecule at D. All species are then assumed to mix and react to equilibrium. The equilibrium composition and enthalpy are determined through the use of the NASA Lewis CETPC code. ${ }^{1}$ The momentum contributions are similarly summed, and used to arrive at a mixture streamwise velocity. Also noted on Fig. 2 is the streamwise convection distance of the resulting equilibrium composition, given by

$$
x=\bar{U} \times I
$$

where the average of the two freestream velocities is used.

Inherent in the methodology are many simplifications, necessary to provide the code with its desired simplicity and speed. One simplification is the time-averaged nature of the calculation of contributions. This description assumes that all elements at the streamwise calculation location contribute to the element of interest at all times. Thus, the time-dependent nature of turbulent mixing is not modeled. It is also assumed that upon reaching the central element, all contributions mix well to equilibrium. To provide a correction for these two assumptions, an efficiency term is introduced. This idea of a time-averaged efficiency was developed while observing Laser Induced Fluorescence (LIF) images of the reacting shear layer. It was noted that combustion 
products were present in small pockets, or eddies, sporadically throughout the mixing layer. By extending the physical observations to a time averaging of post-combustion temperature and freestream temperatures, the experimentally observed thermal profiles for the reacting shear layer were very closely reproduced.

This efficiency term is used as the fraction of time that the calculated molecular distributions are indeed present, and react to equilibrium. For the remaining time, each of the contributing elements is assumed to be present according to its statistically weighted contribution. The calculated temperature, then, is a time average of the reacted mix and the component elements. The calculated width of the reacted element is corrected by the ratio of the averaged and post-reaction temperatures. Following convection, subsequent mixing calculations are based on the equilibrium composition. In the results presented herein, an efficiency of 80 percent has been used. This value produces an approximate match between the peak experimental and calculated temperatures.

Due to the nature of the computational method, profiles are generated only at certain streamwise positions as determined by the product of average streamwise velocity and local integral time scale I. Experimental data, too, exists only at specific streamwise positions. To allow comparisons between experimental and computational results, an interpolation routine for the calculated flowfield was developed to provide profiles at desired streamwise positions. For the non-reacting case, this consisted of non-dimensionalizing the transverse velocity and thermal profiles into two curves, both similar to the error function. These non-dimensionalized profiles were found to be self-similar at all streamwise locations, allowing interpolation at intermediate stations. Linear fits to transverse width and centerline position were used to determine required fitting parameters at any desired streamwise position, and profiles generated. For the reacting case, the additional complication of heat release is present. It was found that by modeling a non-reacting thermal layer after the non-dimensional velocity layer, fitting a non-reacting thermal profile, and subtracting it from the computed reacting thermal profiles, a somewhat bell-shaped curve of temperature rise was formed. Non-dimensionalizing this curve by its maximum value led to a self-similar family of non-dimensional temperature rise curves for all streamwise positions. By fitting lines to the maximum temperature rise, its transverse position, and the temperature rise width as a function of streamwise distance, reacting thermal profiles were generated at desired streamwise locations.

Throughout model development, a measure of the growth rate of the calculated mixing layers was needed for comparison with experimental data. The 10 percent delta thicknesses $b_{T}$ and $b_{V}$ were used for thermal and velocity thicknesses respectively. The upper and lower bounds of the mixing layer are denoted as the point where the local condition departs from the near freestream condition by 10 percent of the freestream velocity or temperature differential. The distance between these two bounds is the local width $b$ of the mixing layer. The growth rate is the width $b$ divided by the streamwise distance $X$ from the splitter plate tip, as

$$
\frac{d b}{d x}=\frac{b}{X}
$$

\section{COMPUTATIONAL PARAMETERS}

The functional form for the integral time scale $I$ was chosen through comparison of experimental growth rates with computational results. It was determined that a constant value of $I$ produced a narrow computational mixing layer. To obtain growth rates similar to those observed experimentally, the time scale had to increase with downstream distance. A linearly increasing time scale is in accordance with intuitive expectations. The effect of the slope of $I$ on mixing layer growth rate was investigated for a linear formulation. For the nonreacting case, the mixing layer velocity width $b_{V}$ was found to vary as the slope of $I$ raised to the $1 / 4$ power. The effect of a large non-zero intercept for the $I$ vs $X$ curve is to provide a rapid growth region at the beginning of the mixing layer. The subsequent growth rate is not affected. For computational purposes, a non-zero 
intercept is required. The intercept used, $0.01 \mathrm{~ms}$, is small enough to provide an effectively zero initial width of the layer.

Experimental determination of the flowfield integral time scales yields varying values in both the streamwise and transverse directions. The integral time scale is the integral of the autocorrelation coefficient, as

$$
I=0 \int^{\infty} \rho(\tau) d \tau
$$

where

$$
\rho(\tau)=\frac{1}{\overline{u^{2}(t)}} \times[u(t) \times u(t+\tau)]
$$

In general, values of $I$ peaked within the mixing layer region and increased with downstream distance. The current resolution of experimental data, however, is insufficient to define the form of $I$ in the transverse direction accurately. To keep the computational model simple, it was desired to use a constant value in the cross-stream direction. Maximum experimental values of $I$ divided by the position $X$ of measurement yield a slope of the $I$ vs $X$ curve of the order of $0.006 \mathrm{~s} / \mathrm{m}$; this is the value used throughout all simulations. Use of the smaller $I$ values present in the freestream results in a narrow computational layer. Insertion of a transverse $I$ profile in keeping with experimental observation would have introduced undesirable complications into the model.

As mentioned in the Computational Model section, a gaussian PDF was used to describe the transverse turbulence profile. Analysis of experimental data $\mathrm{a}^{3,4}$ indicates this is a reasonable approximation. As in the case of the integral time scale, actual experimental data for the transverse turbulence PDF varies in both the streamwise and cross-stream directions. Again, undesirable complications arise from the use of non-constant $V_{\text {rms }}^{\prime}$ values. The relationship between the rms value of transverse velocity and the mixing layer growth rate was investigated. The velocity layer growth rate of the non-reacting mixing layer was found to be given approximately by

$$
\frac{b_{V}}{X}=1.93 \times \frac{3 \times V_{\mathrm{rms}}^{\prime}}{\bar{U}}
$$

for the formulation of $I$ discussed previously. A corresponding simple expression for reacting mixing layers is not available due to the expansion that occurs in accordance with heat release. The factor of three in the above expression arises from the gaussian transverse turbulent profile. Three times the root mean square value encompasses 99.7 percent of the area under a gaussian curve, and very little fluid with velocity magnitudes in excess of this figure move throughout the flowfield in this formulation.

By equating the above expression with the experimental non-reacting mixing layer growth rate, a value of $V_{\mathrm{rms}}^{\prime}=14.6 \mathrm{~m} / \mathrm{s}$ was found to reproduce the non-reacting velocity layer growth rate. Experimental values of $V_{\mathrm{rms}}^{\prime}$ non-dimensionalized by average streamwise velocity are shown in Fig. 3 along with the empirically determined computational value. This value is on the high end of experimentally observed transverse velocity $\mathrm{rms}$ values. Use of an average value results in a computational mixing layer more narrow than the experimentally observed result. Thus, as in the case of the integral time scale, the model requires the use of the maximum value present in the flowfield to reproduce the experimental result. 
A value for the Prandtl number, or ratio of momentum transport to thermal transport, was chosen based on the relative widths of computed non-reacting thermal and velocity mixing layers as $2 / 3$. The same value was assumed for the Schmidt number. This value was implemented as a multiplier of the rms transverse velocity value to effect a change in molecular transport.

Table I summarizes flow conditions for the two simulations. In the non-reacting case, pure nitrogen was used to simulate the air stream. The Results section presents comparisons between experimental and computational profiles. Noticeable in many of the figures are discrepancies between computational and experimental freestream flow variables. These arise from the limitations of both experiment and computation. Computationally, we have constant freestream temperatures and velocities. Experimentally, freestream temperatures and velocities tend to drop somewhat with streamwise position. Thus, it is impossible to match freestream conditions at all positions of comparison. Freestream velocities were chosen to nearly match the majority of the data, along with temperatures. Due to this small departure from specified experimental inlet conditions ${ }^{3,4}$, adjustments to the freestream static pressure of the order of one percent were necessary to match the experimentally specified inlet mass flow rates.

The values of $V_{\text {rms }}^{\prime}, I$, and turbulent Schmidt number $S_{c}$ determined as previously discussed for the nonreacting mixing layer were applied to both flowfields. These are the most significant variables in determining mixing layer growth rate. The large differences in growth rate observed computationally and presented in the results section are due to the expansion which occurs under the constant pressure combustion for the reacting mixing layer.

\section{RESULTS}

Figure 4 shows the calculated mixing layer temperature and velocity contours for the reacting shear layer simulation. Due to the positive mean transverse velocity, the mixing layer moves upward in the duct. The mixing layer widths increase linearly with streamwise distance. Similar flowfield visualizations for the non-reacting case show the centerline of the mixing layer to be below the duct midline, in spite of the zero mean transverse velocity. This is due to the momentum and enthalpic mixing of the low density high speed lower stream, and high density low speed upper stream. This observation agrees with the experimentally observed result. As will be seen in data to be presented, the reacting layer is found to be much wider than the non-reacting layer due to the expansion of the constant-pressure combustion products. This, too, is in qualitative agreement with experimental data.

Shown in Fig. 5 is a comparison between experimental and calculated temperatures at a position $150 \mathrm{~mm}$ downstream from the splitter plate tip. For the non-reacting case, the calculation reproduces the growth rate of the experimentally measured thermal mixing layer well. As this case was utilized to determine the Prandtl and Schmidt numbers to use for the simulation, the agreement in shear layer width is expected. The computational profile is centered approximately $2 \mathrm{~mm}$ high relative to the experimental data, however. The Schmidt number determined through the non-reacting thermal width was used in the calculation of the reacting thermal profile, also shown in Fig. 5. The experimental reacting profile is similar in shape to the experimental result. However, the experiment profile shows a thicker region of high temperature gas. As in the non-reacting case, the vertical position of the experimental thermal layer is poorly predicted. The prediction of vertical position of the thermal and velocity mixing layers will be discussed following presentation of all comparisons between experimental and computational data.

Figure 6 shows the temperature profile data $300 \mathrm{~mm}$ downstream from the splitter plate tip. As in the case of the $150 \mathrm{~mm}$ data, good agreement is seen between the computational and experimental results in width of the non-reacting thermal mixing layer. Again, the vertical positioning is not reproduced. For the reacting 
shear layer, the comparison between the computational and experimental result is excellent. The general shape of the thermal layer, as well as the width of the high temperature region, is well-matched.

Figure 7 shows the calculated and experimentally obtained velocity profiles for non-reacting flow. While the vertical positioning of the velocity mixing layer is again poorly modeled, the diffusion is closely matched for both the $X=150 \mathrm{~mm}$ and $X=300 \mathrm{~mm}$ streamwise positions. As in the case of the non-reacting temperature profile width, this agreement is expected due to the empirical nature of the formulation for the non-reacting case. The non-reacting mixing layer widths were used in conjunction with the growth rate formula presented previously to arrive at the value of the transverse turbulent root mean square velocity used in computations.

Applying the values obtained through the non-reacting studies, the model produces the reacting layer velocity profiles shown in Fig. 8. Here, we see excellent agreement between experimentally measured velocity profiles and the computational result for shear layer width at both positions of comparison. The $X=150 \mathrm{~mm}$ streamwise position shows a slight discrepancy in the vertical placement of the mixing layer between the computational and experimental result. The $X=300 \mathrm{~mm}$ data, however, is well-modeled both in layer width and vertical position.

\section{DISCUSSION}

As mentioned previously in presentation of the computational results, the good agreement between computed and measured thermal and velocity mixing layer widths is expected for the non-reacting simulations. This is due to the empirical method used to arrive at a formulation of $I$, the value of the rms transverse velocity, and the Schmidt number. These three quantities are the significant flowfield variables in control of mixing layer width in this model. The test of the method lies in whether the shapes of the non-reacting profiles are matched, and whether the flowfield parameters obtained through analysis of non-reacting mixing layers can successfully model the reacting shear layer.

For evaluation of the computational work with the experimental data, there are three comparisons available. One is the general shape of the contours. The experimental non-reacting temperature data, and velocimetry data from both reacting and non-reacting cases, has been shown ${ }^{3,4}$ to collapse very nicely onto an error function curve. The calculated profiles differ slightly from the error function due to the momentum and enthalpic mixing. Collapsed thermal and velocity non-reacting profiles are shown in Fig. 9, along with the error function. If simple averages are used of temperatures or velocities, the gaussian mixing used will generate error function profiles as a result. However, the weighting afforded by streams of differing densities and enthalpies results in computational profiles that differ somewhat from that of the error function. This points out a limitation of the computational method; the shapes of the profiles generated are fundamentally somewhat different than those observed experimentally. This limitation is observable in the computational profiles presented previously as a more gentle corner on the upper side of the velocity profiles and non-reacting thermal profiles as compared to the experimental data. The discrepancy, however, is not large.

A second criterion is the modeling of diffusion rate for the shear layer with heat release. Looking at the comparisons between widths of experimental and computational velocity layers, we see that the growth rates are very well modeled with the assumed integral time scale and transverse velocity PDF for the reacting mixing layer. As mentioned previously, the large relative velocity width $b_{V}$ of the reacting mixing layer relative to the non-reacting case is due entirely to thermal expansion in the reacting case. All flow variables which contribute significantly to growth rate were held constant between the two cases. The thermal layer at $300 \mathrm{~mm}$ downstream of the splitter plate is well modeled in shape and width. From these comparisons, it appears that the diffusion, heat release, and subsequent layer expansion is well modeled. 
The discrepancy between computational and experimental $150 \mathrm{~mm}$ thermal data is difficult to explain with this in mind. The shape of the reacting thermal layer here seems to be the problem as much as the width. Experimentally, the mixing layer region appears to have expanded to a larger degree than in the computational result. An investigation of the effect of Schmidt number and efficiency on the reacting thermal layer shape provided no means of matching the observed profile. Simulation of a higher diffusion rate, through use of a smaller Schmidt number, tends to increase the width of the wings of the calculated layer more than the middle. Increasing the efficiency increases heat release, and so results in greater expansion; however, the resulting temperature increase at all points in the flowfield results in a computational profile that simply overshoots the experimental data everywhere. Thus, at this time, the discrepancy is unexplained. Further examination of additional experimental thermocouple data may shed some light on the problem.

A third criterion is the vertical placement of the mixing layer, which is rather poorly modeled in many cases. This is to be expected to a certain degree. The code is incapable of simulating any fluid dynamic effects which might result in curvature of the mixing layer, or movement in any other than a constant angle with respect to test section centerline. However, the mismatch may be partially attributed to limitations of the experimental data. The experimental thermal profiles presented for a given condition were obtained on different dates, and none in conjunction with the velocimetry data presented. Thus, the vertical placement, controlled through the mean transverse velocity, is effectively a guess for the thermal profiles. Data has shown that the mixing layer position in the vertical direction changes considerably from test to test. The phenomenon behind this placement of the layer is not yet understood. The current feeling is that non-zero mean vertical velocities are the cause; but, the source of the non-zero mean velocities is not yet understood. The vitiating torch ${ }^{3}$ used to ignite the mixing layer is one possible source of the vertical velocity component. Inconsistencies between mixing layer position in the transverse direction at the two streamwise positions are therefore not regarded as a serious limitation of the computational method at this time. Further experimental investigations will continue to define the computational boundary conditions.

The discrepancies in the position of the velocity mixing layer, too, are not regarded as a serious deficiency. It has been determined that the test section moves several millimeters both vertically and horizontally during testing due to thermal expansion. Feedback sensors to account for this expansion have recently been installed on the test rig. Additional data with the origin corrected should remove any ambiguity from the vertical and horizontal distance between the splitter plate tip and the measurement position, allowing continued code evaluation and refinement.

The success of the code in simulating both the non-reacting and reacting thermal layers through simulation of molecular transport is due to the fact that large scale movements of gases are allowed in the model. The large influence distance discussed previously allows reactants to penetrate deeply into the computational mixing layer, resulting in a large heat release. The success of the model indicates that the simulation of large-scale transport of molecules is necessary to reproduce this highly turbulent flowfield. Molecular diffusion, as opposed to turbulent mixing, is not modeled here except in the form of the inefficiency discussed previously. Increasing the efficiency, and hence gaseous diffusion rates, does not reproduce the wide thermal layers without the mixing-based handling of the Schmidt number.

\section{CODE APPLICATION}

The results presented herein are the only ones generated to date by this code. To keep the code as general and simple as possible, the values of the root mean square transverse turbulent velocity and the integral time scale were held constant throughout the flowfield. With these simplifications, it was found that the use of the maximum values of turbulence parameters present in the shear layer was necessary to reproduce the experi- 
mental thermal and velocity profiles. A desirable extension of the present work is to apply the code to alternate geometries and flow conditions, and evaluate the performance.

Suitable for testing are high Reynolds number annular or planar mixing layers. Recommended flowfield variables, based on the current result, are a root mean square transverse velocity equal to 5.7 percent of the mean freestream velocity; a turbulent Schmidt number of 2/3; and an integral time scale formulation of $I=0.00001+0.006 * X$ in units of seconds, with streamwise distance $X$ in meters. A mixing and combustion efficiency of 80 percent could be used initially, with correction made to match peak experimental temperatures if such data were available.

\section{CONCLUSIONS}

A simple methodology has been developed for the simulation of the PRSL facility reacting flowfields. Mixing rates between the two streams are simulated relatively accurately, with the momentum mixing fitting experimental data more closely than the thermal. Mixing layer centerline position and curvature are not well modeled, but the discrepancies are not viewed as serious limitations of the model at this time due to experimental uncertainties. The simulations suggest a mixing layer efficiency of approximately 80 percent and a turbulent Schmidt number of $2 / 3$. Identical turbulent flowfield variables for both cases successfully reproduce experimental data, suggesting that the fundamental properties of the flowfields are similar. The success of the model indicates that simulation of large-scale transport is necessary to reproduce the observed properties of this highly turbulent mixing layer. The calculation is simple enough to run in a few minutes on a desktop computer, and shows that a simple model based on bulk transverse turbulent transport can effectively reproduce thermal and velocity experimental data for this facility. The formulation has not yet been applied to any other facility or flow geometries, and should be compared with additional experimental data before it is applied to an untested flowfield to generate profiles.

\section{ACKNOWLEDGMENTS}

The author is indebted to Dr. C. John Marek for many insightful comments and helpful discussions throughout the course of model development, and to the NASA Lewis Aerothermochemistry Branch for support-

ing this research. Ms. Bonnie McBride was extremely helpful regarding the use of the CETPC equilibrium chemistry code. Professor H.T. Nagamatsu and Professor L.N. Myrabo contributed throughout.

\section{REFERENCES}

1. Gordon, S.; and McBride, B.J.: Computer Program for Calculation of Complex Chemical Equilibrium Compositions, Rocket Performance, Incident and Reflected Shocks, and Chapman-Jouget Detonations. NASA SP-273, 1976.

2. Marek, C.J., et al.: A Planar Reacting Shear Layer System for the Study of Fluid Dynamics-Combustion Interaction. NASA TM-102422, 1990.

3. Chang, C.T., et al.: Comparison of Reacting and Non-Reacting Shear Layers at a High Subsonic Mach Number. AIAA Paper 93-2381, 1993. (Also, NASA TM-106198, 1993.)

4. Chang, C.T., et al.: Turbulence Measurement in a Reacting and Non-reacting Shear Layer at a High Subsonic Mach Number. NASA TM-106186, 1993. 
5. Konrad, J.H.: An Experimental Investigation of Mixing in Two-Dimensional Turbulent Shear Flows with Applications to Diffusion-Limited Chemical Reactions. Project Squid Technical Report CIT-8-PU, Project Squid Headquarters, Purdue University, West Lafayette, IN, Dec. 1976. (Also, Ph.D. Thesis, California Institute of Technology, Pasadena, CA, 1977.)

6. Wallace, A.K.: Experimental Investigation on the Effects of Chemical Heat Release in the Reacting Tubulent Plane Shear Layer. AFOSR Technical Report 84-0650, Bolling Air Force Base, Washington DC, Jan. 1981. (Also, Ph.D. Thesis, California Institute of Technology, Pasadena, CA, 1981.)

7. McBride, B.J.; Gordon, S.; and Reno, M.A.: Thermodynamic Data for Fifty Reference Elements. NASA TP-3287, 1993.

TABLE I.-FLOW CONDITIONS AND STATISTICAL DATA FOR SIMULATIONS

\begin{tabular}{|l|r|r|}
\hline \multicolumn{1}{|c|}{ Input Values } & Non-Reacting Flow & \multicolumn{1}{c|}{ Reacting Flow } \\
\hline Lower duct air flow, $\mathrm{kg} / \mathrm{s}$ & 1.87 & 1.94 \\
\hline Upper duct $\mathrm{N}_{2}$ flow, $\mathrm{kg} / \mathrm{s}$ & 1.62 & 1.00 \\
\hline Upper duct $\mathrm{H}_{2}$ flow, $\mathrm{kg} / \mathrm{s}$ & 0.000 & 0.032 \\
\hline Lower duct velocity, $\mathrm{m} / \mathrm{s}$ & 380 & 375 \\
\hline Upper duct velocity, $\mathrm{m} / \mathrm{s}$ & 134 & 135 \\
\hline Lower duct static temp., $\mathrm{K}$ & 758 & 740 \\
\hline Upper duct static temp., $\mathrm{K}$ & 335 & 322 \\
\hline Static pressure, Pa & 100,000 & 106,300 \\
\hline Avg. transverse velocity, $\mathrm{m} / \mathrm{s}$ & 0 & 12 \\
\hline RMS transverse velocity, $\mathrm{m} / \mathrm{s}$ & 14.6 & 14.6 \\
\hline Integral time scale, $\mathrm{s}(\mathrm{X} \mathrm{in} \mathrm{m})$ & $0.00001+0.006 * \mathrm{X}$ & $0.00001+0.006 * \mathrm{X}$ \\
\hline
\end{tabular}

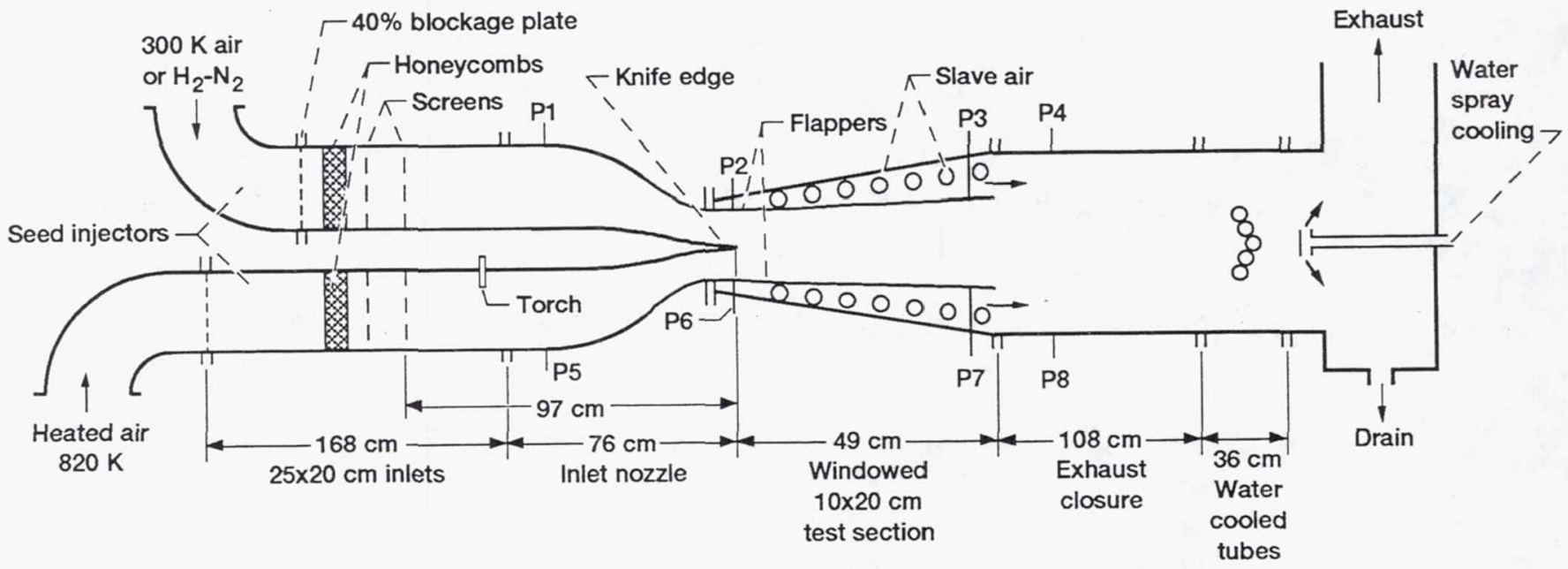

Figure 1.- Planar reacting shear layer facility schematic. 


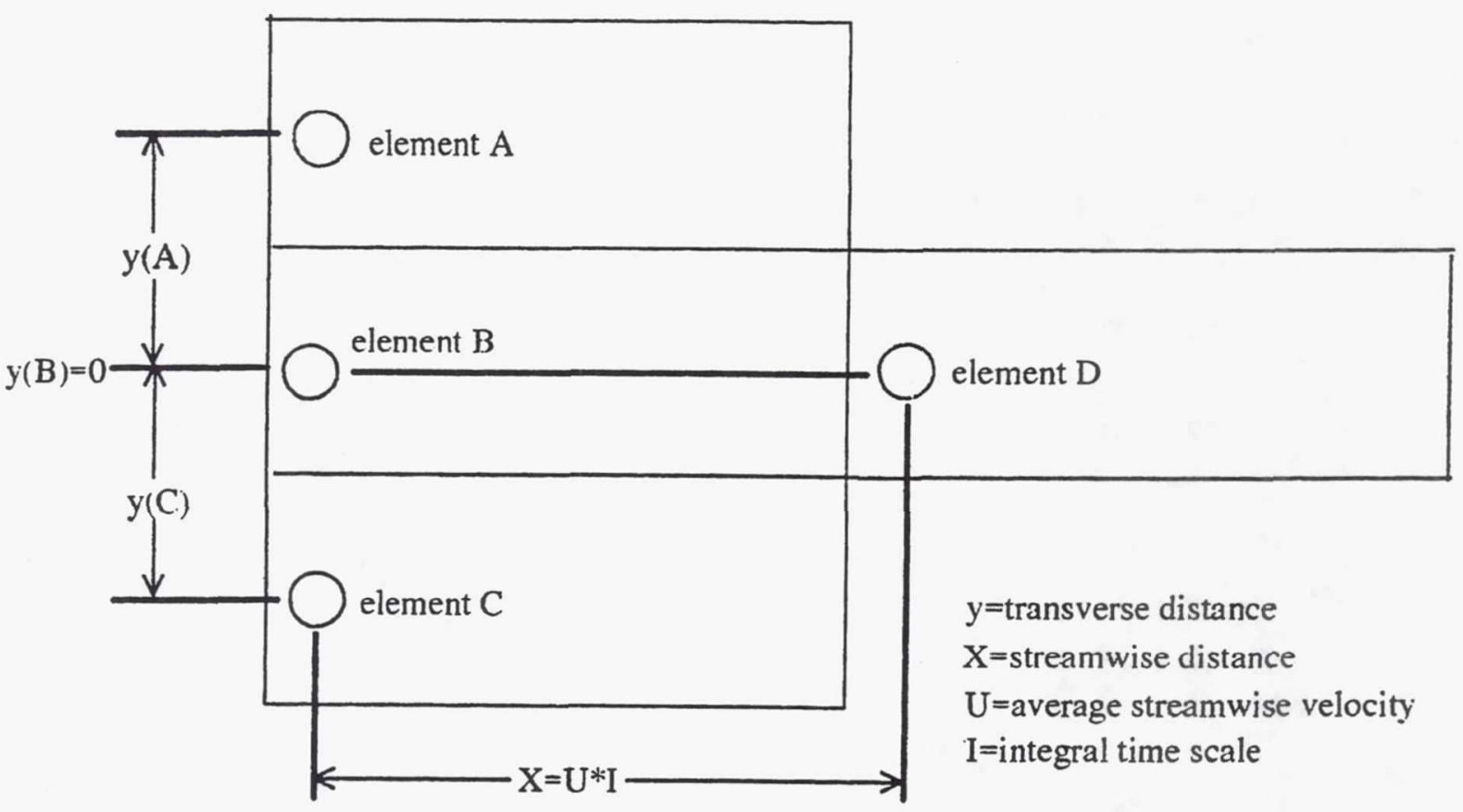

Figure 2.-Computational fluid elements and transport distances.

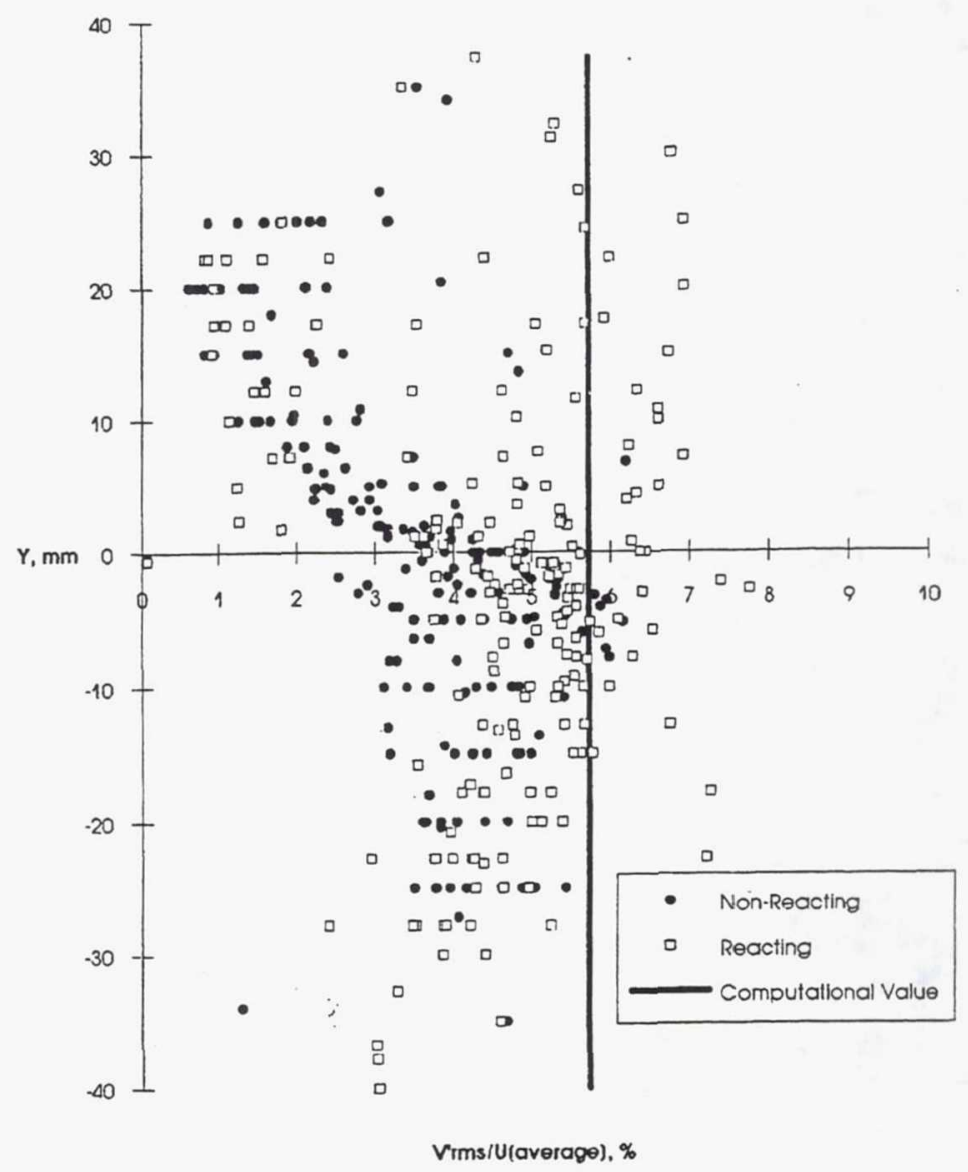

Figure 3.-Computational and experimental transverse turbulent velocity RMS values. 


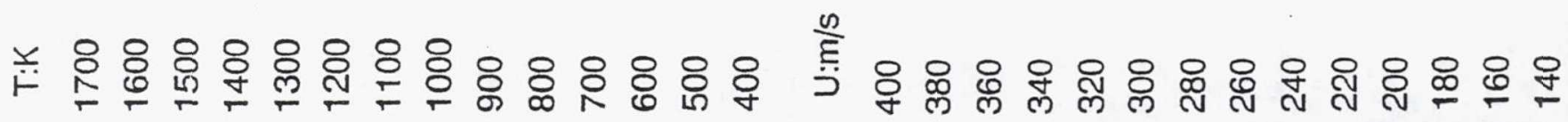



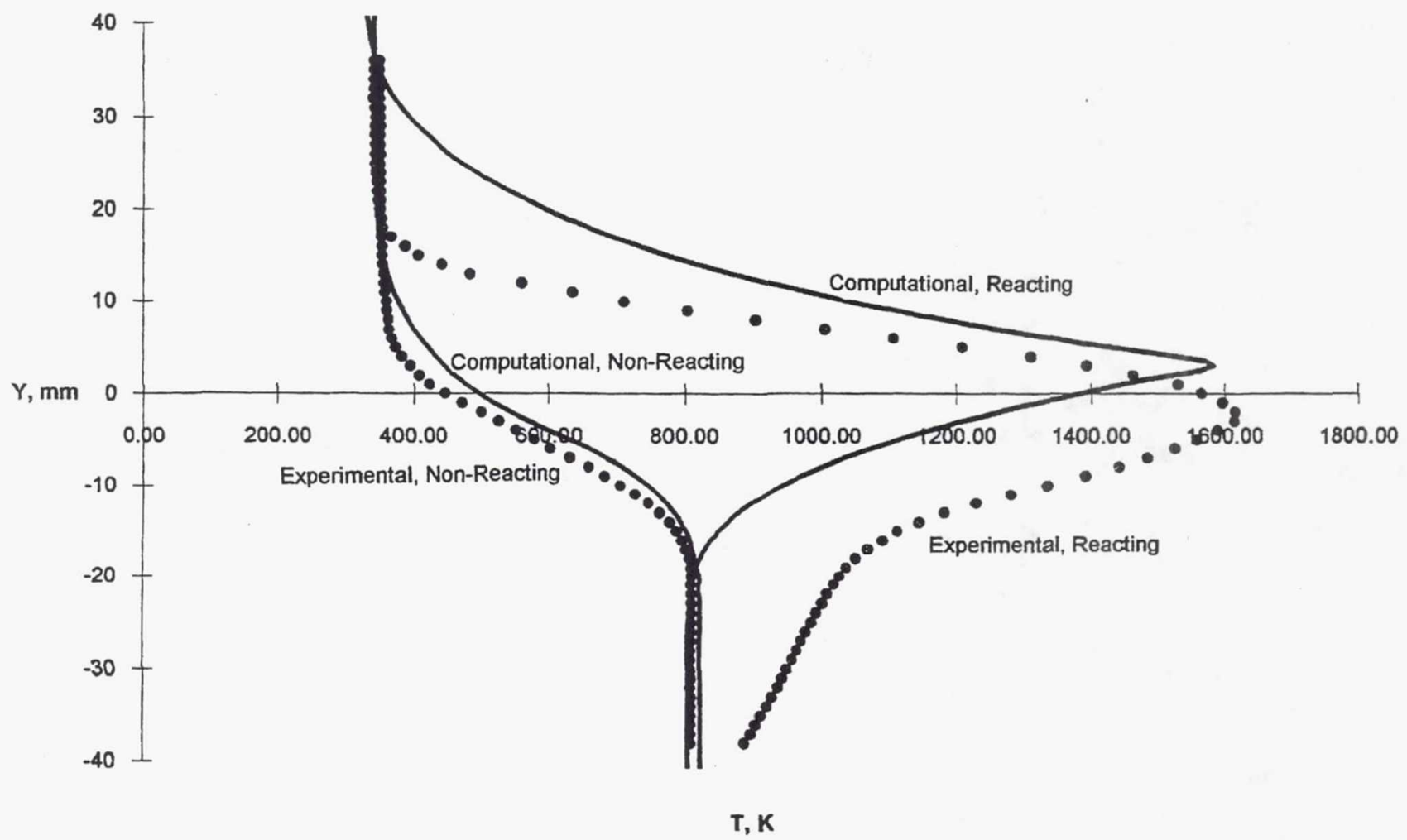

Figure 5.-Computational and experimental thermal profiles, $X=150 \mathrm{~mm}$.

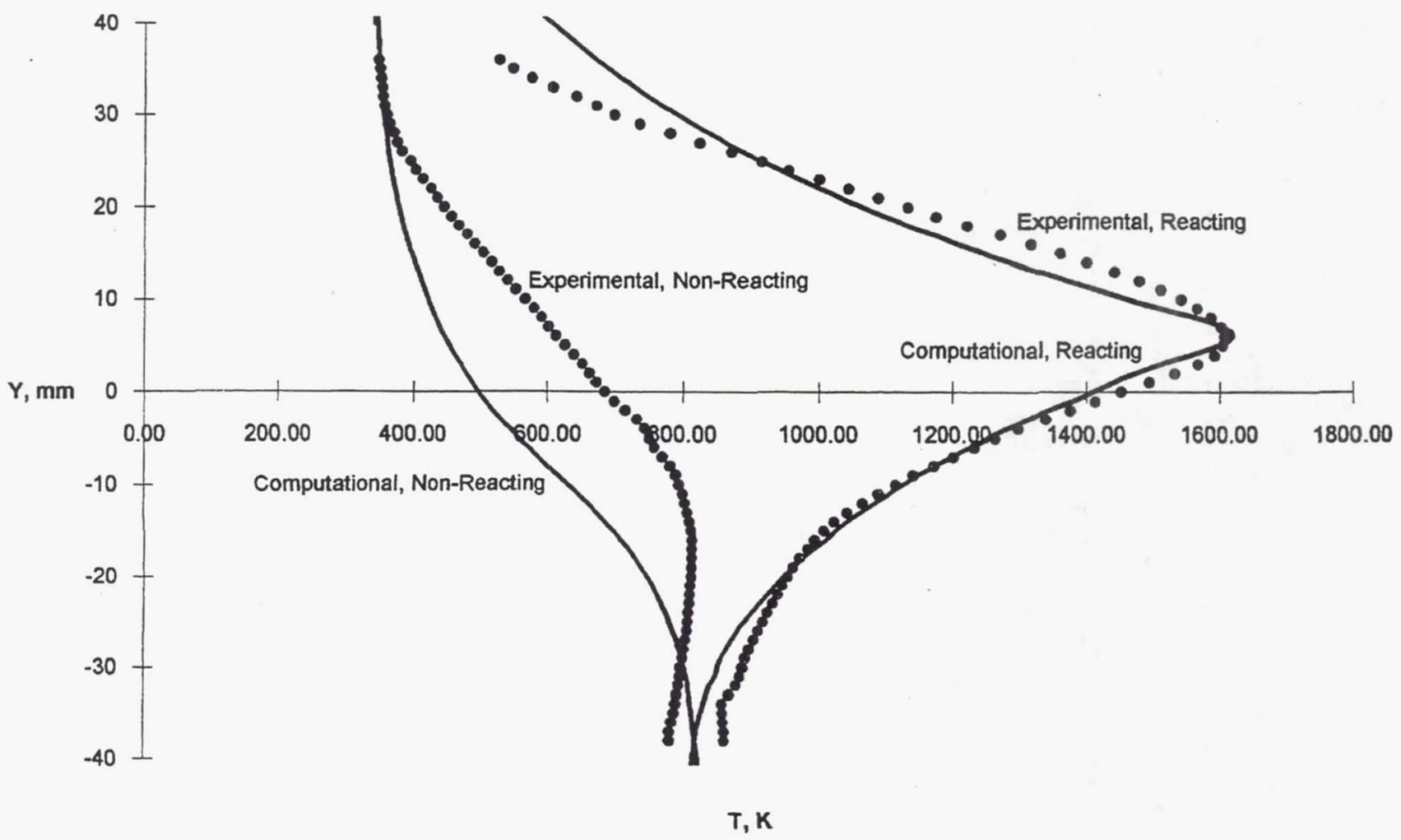

Figure 6.-Computational and experimental thermal profiles, $X=300 \mathrm{~mm}$. 


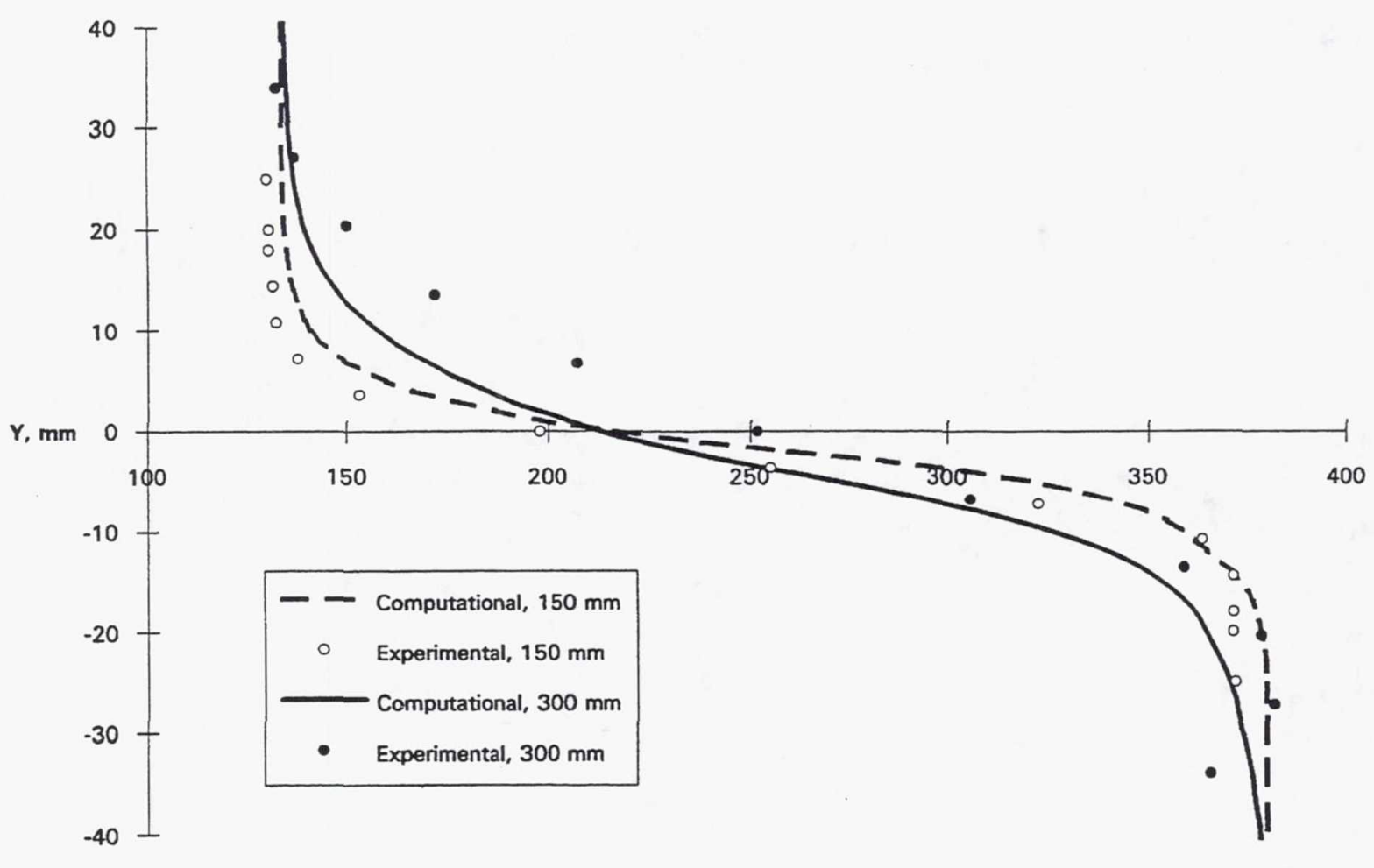

$\mathrm{U}, \mathrm{m} / \mathrm{s}$

Figure 7.-Computational and experimental non-reacting layer velocity profiles.

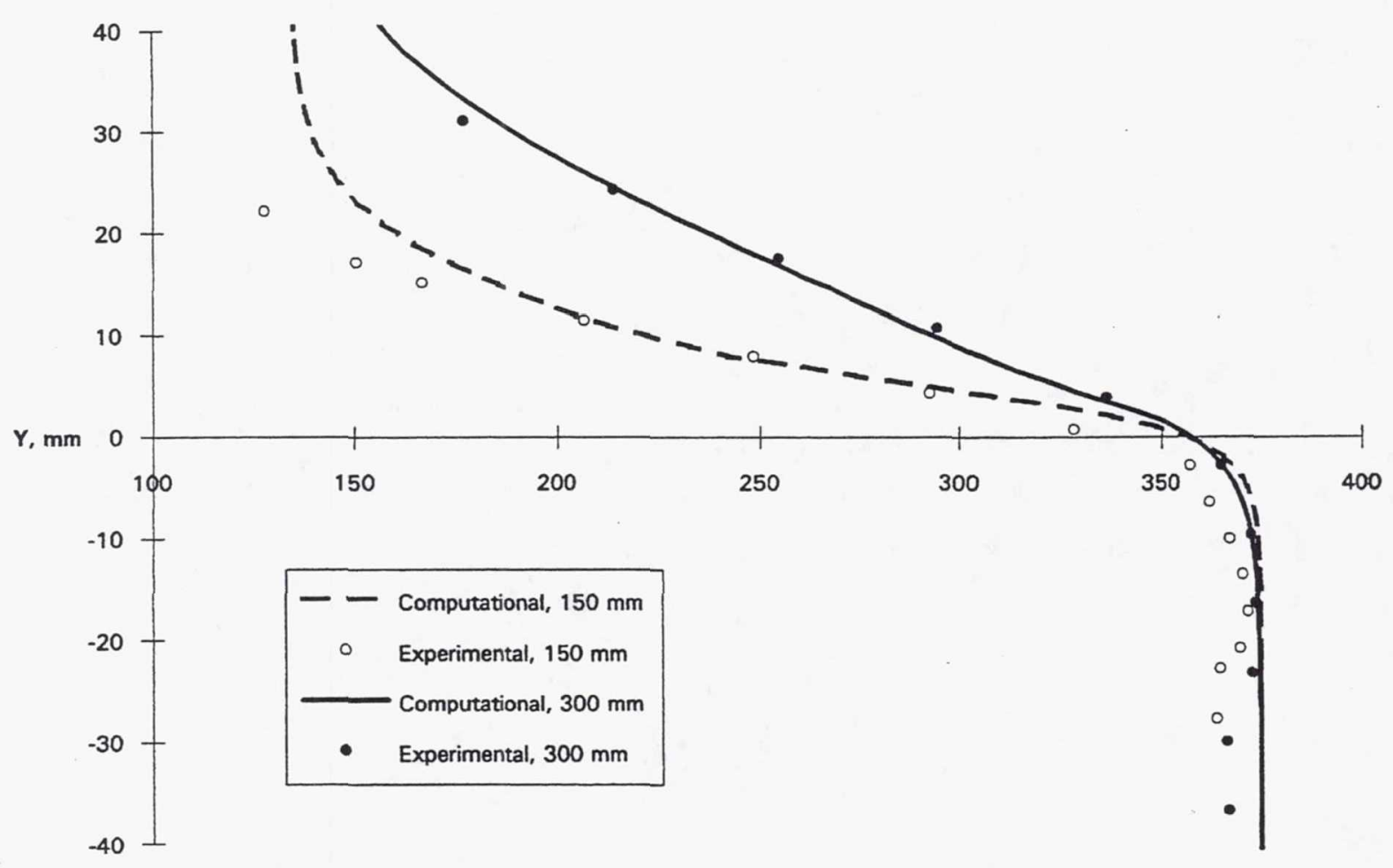

$\mathrm{U}, \mathrm{m} / \mathrm{s}$

Figure 8.-Computational and experimental reacting layer velocity profiles. 


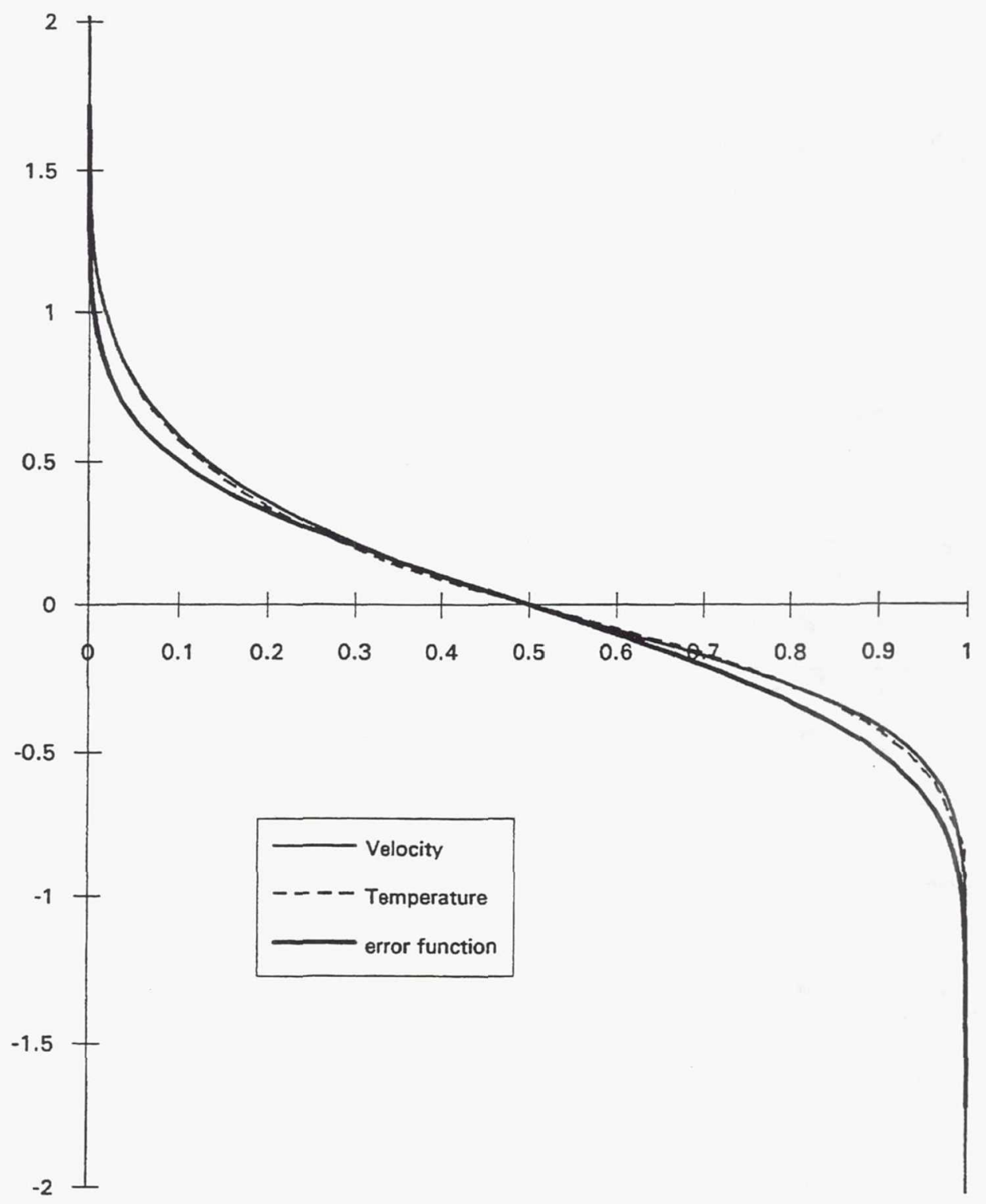

Figure 9.-Computational non-dimensionalized profiles and the error function. 
Public reporting burden for this collection of information is estimated to average 1 hour per response, including the time for reviewing instructions, searching existing data sources, gathering and maintaining the data needed, and completing and reviewing the collection of information. Send comments regarding this burden estimate or any other aspect of this Davis Highway, Suite 1204, Arlington, VA 22202-4302, and to the Office of Management and Budget, Paperwork Reduction Project (0704-0188), Washington, DC 20503.

\begin{tabular}{|l|l|l}
\hline 1. AGENCY USE ONLY (Leave blank) & $\begin{array}{c}\text { 2. REPORT DATE } \\
\text { January } 1994\end{array}$ & $\begin{array}{r}\text { 3. REPORT TYPE AND DATES COVERED } \\
\text { Final Contractor Report }\end{array}$ \\
\hline
\end{tabular}

4. TITLE AND SUBTITLE 5. FUNDING NUMBERS

A Random Distribution Reacting Mixing Layer Model

6. AUTHOR(S)

Richard A. Jones

WU-505-62-52

NCC-3-213

7. PERFORMING ORGANIZATION NAME(S) AND ADDRESS(ES)

8. PERFORMING ORGANIZATION REPORT NUMBER

Rensselaer Polytechnic Institute

Troy, New York 12181 AGENCY REPORT NUMBER

National Aeronautics and Space Administration

Lewis Research Center

NASA CR-194445

Cleveland, Ohio 44135-3191

\section{SUPPLEMENTARY NOTES}

Richard A. Jones, Rensselaer Polytechnic Institute, Troy, New York 12181 and Summer Student Intern at NASA Lewis Research Center. Project Manager, C.J. Marek, Internal Fluid Mechanics Division, (216) 433-3584.

12a. DISTRIBUTION/AVAILABILITY STATEMENT

12b. DISTRIBUTION CODE

Unclassified - Unlimited

Subject Category 07

13. ABSTRACT (Maximum 200 words)

A methodology for simulation of molecular mixing, and the resulting velocity and temperature fields has been developed. The ideas are applied to the flow conditions present in the NASA Lewis Planar Reacting Shear Layer (PRSL) facility, and results compared to experimental data. A gaussian transverse turbulent velocity distribution is used in conjunction with a linearly increasing time scale to describe the mixing of different regions of the flow. Equilibrium reaction calculations are then performed on the mix to arrive at a new species composition and temperature. Velocities are determined through summation of momentum contributions. The analysis indicates a combustion efficiency of the order of 80 percent for the reacting mixing layer, and a turbulent Schmidt number of $2 / 3$. The success of the model is attributed to the simulation of large-scale transport of fluid. The favorable comparison shows that a relatively quick and simple PC calculation is capable of simulating the basic flow structure in the reacting and non-reacting shear layer present in the facility given basic assumptions about turbulence properties.

14. SUBJECT TERMS

Turbulent combustion; Gaussian distribution; Shear layer; Random

\begin{tabular}{|c|c|c|}
\hline $\begin{array}{c}\text { 17. SECURITY CLASSIFICATION } \\
\text { OF REPORT } \\
\text { Unclassified }\end{array}$ & $\begin{array}{c}\text { 18. SECURITY CLASSIFICATION } \\
\text { OF THIS PAGE } \\
\text { Unclassified }\end{array}$ & $\begin{array}{c}\text { 19. SECURITY CLASSIFICATION } \\
\text { OF ABSTRACT } \\
\text { Unclassified }\end{array}$ \\
\hline
\end{tabular}




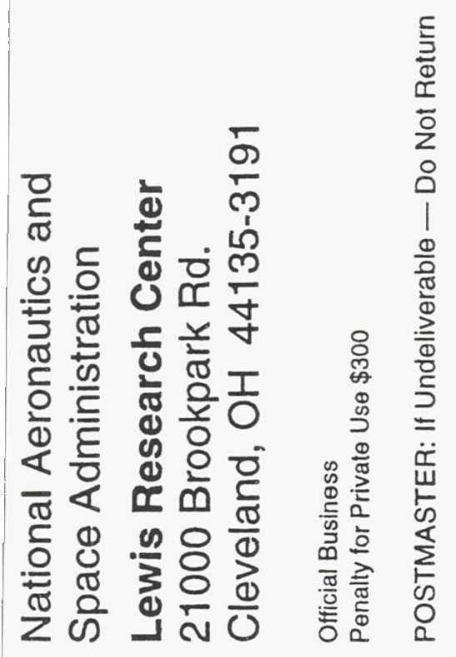

